\title{
THE COMPLEX ZEROS OF RANDOM POLYNOMIALS
}

\author{
LARRY A. SHEPP AND ROBERT J. VANDERBEI
}

ABSTRACT. Mark Kac gave an explicit formula for the expectation of the number, $\nu_{n}(\Omega)$, of zeros of a random polynomial,

$$
P_{n}(z)=\sum_{j=0}^{n-1} \eta_{j} z^{j},
$$

in any measurablc subset $\Omega$ of the reals. Here, $\eta_{0}, \ldots, \eta_{n-1}$ are independent standard normal random variables. In fact, for each $n>1$, he obtained an explicit intensity function $g_{n}$ for which

$$
\mathbf{E} \nu_{n}(\Omega)=\int_{\Omega} g_{n}(x) d x .
$$

Here, we extend this formula to obtain an explicit formula for the expected number of zeros in any measurable subset $\Omega$ of the complex plane $\mathbb{C}$. Namely, we show that

$$
\mathbf{E} \nu_{n}(\Omega)=\int_{\Omega} h_{n}(x, y) d x d y+\int_{\Omega \cap \mathbb{R}} g_{n}(x) d x,
$$

where $h_{n}$ is an explicit intensity function. We also study the asymptotics of $h_{n}$ showing that for large $n$ its mass lies close to, and is uniformly distributed around, the unit circle.

\section{INTRODUCTION}

More than fifty years ago, Mark Kac [7] gave an explicit formula for the expectation of the number, $\nu_{n}(\Omega)$, of zeros of a random polynomial,

$$
P_{n}(z)=\sum_{j=0}^{n-1} \eta_{j} z^{j}, \quad z \in \mathbb{C},
$$

in any measurable subset $\Omega$ of the reals. Here, $\eta_{0}, \ldots, \eta_{n-1}$ are independent standard normal random variables (which Kac argues is the most natural choice for a "typical" polynomial since this distribution is invariant under orthogonal transformations-but of course other choices for the measure are also interesting). In fact, for each $n>1$, he obtained an explicit intensity function $g_{n}$ for

Received by the editors July 28, 1994 and, in revised form, October 13, 1994.

1991 Mathematics Subject Classification. Primary 30C15, Secondary 30B20, 26C10, 60B99.

The second author's research was supported by AFOSR through grant AFOSR-91-0359. 
which

$$
\mathbf{E} \nu_{n}(\Omega)=\int_{\Omega} g_{n}(x) d x .
$$

Here, we extend this result by deriving an explicit formula for the expected number of zeros in any measurable subset $\Omega$ of the complex plane $\mathbb{C}$. Namely, we show that

$$
\mathbf{E} \nu_{n}(\Omega)=\int_{\Omega} h_{n}(x, y) d x d y+\int_{\Omega \cap \mathbb{R}} g_{n}(x) d x,
$$

where $h_{n}$ is an explicit intensity function. (Note that we make the usual identification between a point $z$ of the complex plane and its real and imaginary parts, $x$ and $y$.)

The intensity function $h_{n}$ is conveniently expressed in terms of the following three real-valued functions defined on $\mathbb{C}$

$$
B_{k}(z)=\sum_{j=0}^{n-1} j^{k}|z|^{2 j}, \quad z \in \mathbb{C}, \quad k=0,1,2,
$$

and the following two complex-valued functions

$$
A_{k}(z)=\sum_{j=0}^{n-1} j^{k} z^{2 j}, \quad z \in \mathbb{C}, \quad k=0,1 .
$$

Finally, let

$$
D_{0}(z)=\sqrt{B_{0}^{2}(z)-\left|A_{0}\right|^{2}(z)} .
$$

Our main result is

Theorem 1.1. For each region $\Omega \in \mathbb{C}$,

$$
\mathbf{E} \nu_{n}(\Omega)=\int_{\Omega} h_{n}(x, y) d x d y+\int_{\Omega \cap \mathbb{R}} g_{n}(x) d x,
$$

where

$$
h_{n}=\frac{B_{2} D_{0}^{2}-B_{0}\left(B_{1}^{2}+\left|A_{1}\right|^{2}\right)+B_{1}\left(A_{0} \overline{A_{1}}+\bar{A}_{0} A_{1}\right)}{\pi|z|^{2} D_{0}^{3}}
$$

and

$$
g_{n}=\frac{\left(B_{0} B_{2}-B_{1}^{2}\right)^{1 / 2}}{\pi|z| B_{0}} .
$$

As already mentioned, $\mathrm{Kac}$ [7] was the first to study the real zeros for random polynomials of this type and he obtained the intensity function $g_{n}$. Somewhat earlier (but delayed in publication by the war), Rice [12] obtained a similar formula for the expected number of zeros of a general stochastic process $X(t)$ again depending on a real parameter $t$ but did not consider the special case when $X$ is a polynomial in $t$. Here, we obtain Kac's $g_{n}$ as a consequence of our analysis of the zeros in the complex plane.

While the definition of $h_{n}$ in Theorem 1.1 looks rather complicated, it is nevertheless amenable both to computation (see Figures 1 to 6 in Section 5) and to asymptotic analysis. Indeed, the computed plots appearing on the lefthand side in Figures 1 to 6 clearly show that as $n$ gets large the zeros tend 
to lie very close to the unit circle and, ignoring the real roots, appear to be approximately uniformly distributed around the circle. Regarding analysis, the next theorem shows that the intensities $h_{n}$ and $g_{n}$ have well-defined limits as $n$ tends to infinity. These limits are best expressed in terms of the following functions:

$$
\begin{aligned}
& B(z)=\frac{1}{1-|z|^{2}}, \\
& A(z)=\frac{1}{1-z^{2}},
\end{aligned}
$$

and

$$
D(z)=\sqrt{B^{2}(z)-|A|^{2}(z)} .
$$

Theorem 1.2. We have the following limits:

$$
h=\lim _{n} h_{n}=\frac{|B| D}{\pi}
$$

and

$$
g=\lim _{n} g_{n}=\frac{1}{\pi}|B| .
$$

The limit functions, $h$ and $g$, can be viewed as intensity functions for the zeros of the random power series

$$
P(z)=\sum_{j=0}^{\infty} \eta_{j} z^{j}
$$

(at least within its radius of convergence, $|z|<1$ ).

Following the initial works of $\mathrm{Kac}$ and Rice, a large body of research on zeros of random polynomials has appeared-see [2] for a fairly complete account including an extensive list of references. Most of this work has focused on the real zeros, although [4], [5] and [14] are a few notable exceptions. Also, the recent paper of Edelman and Kostlan [3] gives a very elegent geometric treatment of the problem.

In the following section, we derive explicit formulas for the intensity functions $h_{n}$ and $g_{n}$. Then in Section 3, we study the limiting form of these functions as $n$ tends to infinity. Section 4 presents more delicate asymptotic analysis that shows how $h_{n}$ and $g_{n}$ behave when $n$ is large but finite. Next, in Section 5 we discuss computational issues, and finally, in Section 6 we offer some speculation and suggest future research directions.

We end this section by remarking that even though we usually consider the entire complex plane, it suffices in general to study simply the unit disk centered at the origin since, given a random polynomial $P_{n}(z)$, the transformation to $z^{n} P_{n}(1 / z)$ produces a new random polynomial whose zeros are exactly the reciprocals of the zeros of $P_{n}(z)$. Hence, for any set $A$, we have that

$$
\mathbf{E} \nu_{n}\left(A^{-1}\right)=\mathbf{E} \nu_{n}(A)
$$


where $A^{-1}=\left\{z: z^{-1} \in A\right\}$. From this it follows easily that any intensity function, say $h$, must satisfy

$$
h(z)=h(1 / z) \frac{1}{|z|^{2}} .
$$

\section{THE INTENSITY FUNCTIONS $h_{n}$ AND $g_{n}$}

This section is devoted to the proof of Theorem 1.1. We begin with

Proposition 2.1. For each region $\Omega \in \mathbb{C}$ whose boundary intersects the real axis at most only finitely many times,

$$
\mathbf{E} \nu_{n}(\Omega)=\frac{1}{2 \pi i} \int_{\partial \Omega} \frac{1}{z} F(z) d z,
$$

where

$$
F=\frac{B_{1} D_{0}+B_{0} B_{1}-\bar{A}_{0} A_{1}}{B_{0} D_{0}+B_{0}^{2}-\bar{A}_{0} A_{0}} .
$$

Proof. The argument principle (see, e.g., [1], p. 151) gives an explicit formula for the random variable $\nu_{n}(\Omega)$, namely

$$
\nu_{n}(\Omega)=\frac{1}{2 \pi i} \int_{\partial \Omega} \frac{P_{n}^{\prime}(z)}{P_{n}(z)} d z .
$$

Taking expectations in (2.3) and then interchanging expectation and contour integration (the justification of which is tedious but doable), we get

$$
\mathbf{E} \nu_{n}(\Omega)=\frac{1}{2 \pi i} \int_{\partial \Omega} \mathbf{E} \frac{P_{n}^{\prime}(z)}{P_{n}(z)} d z .
$$

To facilitate computations, it is advantagous to multiply and divide by $z$ (multiplying inside the expectation and dividing outside). The following lemma shows that away from the real axis the function

$$
F(z)=\mathbf{E} \frac{z P_{n}^{\prime}(z)}{P_{n}(z)}
$$

simplifies to the expression given in $(2.2)$, and since we have assumed that $\partial \Omega$ intersects the real axis at only finitely many points, this finishes the proof.

Lemma 2.2. Let $F$ denote the function defined by (2.5). Off the real axis,

$$
F=\frac{B_{1} D_{0}+B_{0} B_{1}-\bar{A}_{0} A_{1}}{B_{0} D_{0}+B_{0}^{2}-\bar{A}_{0} A_{0}} .
$$

On the real axis, $F$ has a jump discontinuity. Indeed, for each $x \in \mathbb{R}$,

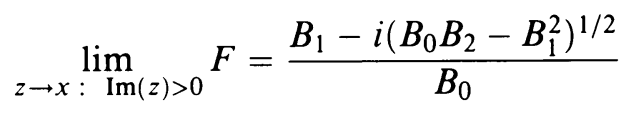

and

$$
\lim _{z \rightarrow x: \operatorname{Im}(z)<0} F=\frac{B_{1}+i\left(B_{0} B_{2}-B_{1}^{2}\right)^{1 / 2}}{B_{0}} .
$$


Proof. First we consider the case where $z$ does not lie on the real axis. Note that $P_{n}(z)$ and $z P_{n}^{\prime}(z)$ are complex Gaussian random variables. It is convenient to work with their real and imaginary parts,

$$
\begin{aligned}
P_{n}(z) & =\xi_{1}+i \xi_{2}, \\
z P_{n}^{\prime}(z) & =\xi_{3}+i \xi_{4},
\end{aligned}
$$

which are just linear combinations of the original standard normal random variables:

$$
\begin{array}{ll}
\xi_{1}=\sum_{j=0}^{n-1} a_{j} \eta_{j}, & \xi_{2}=\sum_{j=0}^{n-1} b_{j} \eta_{j}, \\
\xi_{3}=\sum_{j=0}^{n-1} c_{j} \eta_{j}, & \xi_{4}=\sum_{j=0}^{n-1} d_{j} \eta_{j} .
\end{array}
$$

The coefficients in these linear combinations are given by

$$
\begin{aligned}
a_{j} & =\operatorname{Re}\left(z^{j}\right)=\frac{z^{j}+\bar{z}^{j}}{2}, \\
b_{j} & =\operatorname{Im}\left(z^{j}\right)=\frac{z^{j}-\bar{z}^{j}}{2 i}, \\
c_{j} & =j a_{j}, \\
d_{j} & =j b_{j} .
\end{aligned}
$$

Put $\xi=\left[\begin{array}{llll}\xi_{1} & \xi_{2} & \xi_{3} & \xi_{4}\end{array}\right]^{T}$. The covariance among these four Gaussian random variables is easy to compute:

$$
\operatorname{Cov}(\xi)=\mathbf{E} \xi \xi^{T}=\left[\begin{array}{llll}
a^{T} a & a^{T} b & a^{T} c & a^{T} d \\
b^{T} a & b^{T} b & b^{T} c & b^{T} d \\
c^{T} a & c^{T} b & c^{T} c & c^{T} d \\
d^{T} a & d^{T} b & d^{T} c & d^{T} d
\end{array}\right]
$$

We now represent these four correlated Gaussian random variables in terms of four standard normals. To this end, we seek a lower triangular matrix $L=\left[l_{i j}\right]$ such that the vector $\xi$ is equal in distribution to $L \zeta$, where $\zeta=$ $\left[\begin{array}{llll}\zeta_{1} & \zeta_{2} & \zeta_{3} & \zeta_{4}\end{array}\right]^{T}$ is a vector of four independent standard normal random variables. The following simple calculation shows that $L$ is the Cholesky factor for the covariance matrix:

$$
\operatorname{Cov}(\xi)=\mathbf{E} \xi \xi^{T}=\mathbf{E} L \zeta \zeta^{T} L^{T}=L L^{T} .
$$

Now, since $\xi \stackrel{\mathrm{D}}{=} L \zeta$ and $L$ is lower triangular (the symbol $\stackrel{\mathrm{D}}{=}$ denotes equality in distribution), we get that

$$
\frac{z P_{n}^{\prime}(z)}{P_{n}(z)}=\frac{\xi_{3}+i \xi_{4}}{\xi_{1}+i \xi_{2}} \stackrel{\mathrm{D}}{=} \frac{\left(l_{31}+i l_{41}\right) \zeta_{1}+\left(l_{32}+i l_{42}\right) \zeta_{2}+\left(l_{33}+i l_{43}\right) \zeta_{3}+i l_{44} \zeta_{4}}{\left(l_{11}+i l_{21}\right) \zeta_{1}+i l_{22} \zeta_{2}} .
$$

Hence, exploiting the independence of the $\zeta_{i}$ 's, we see that

$$
F(z)=\mathbf{E} \frac{z P_{n}^{\prime}(z)}{P_{n}(z)}=\mathbf{E} \frac{\alpha \zeta_{1}+\beta \zeta_{2}}{\gamma \zeta_{1}+\delta \zeta_{2}},
$$

where

$$
\begin{array}{rlrl}
\alpha=l_{31}+i l_{41}, & \beta=l_{32}+i l_{42}, \\
\gamma=l_{11}+i l_{21}, & \delta & =i l_{22} .
\end{array}
$$


Splitting up the numerator in (2.11) and exploiting the exchangeability of $\zeta_{1}$ and $\zeta_{2}$, we can rewrite the expectation as

$$
F(z)=\frac{\alpha}{\delta} f(\gamma / \delta)+\frac{\beta}{\gamma} f(\delta / \gamma)
$$

where $f$ is a complex-valued function defined on $\mathbb{C} \backslash \mathbb{R}$ by

$$
f(w)=\mathbf{E} \frac{\zeta_{1}}{w \zeta_{1}+\zeta_{2}}
$$

The expectation that appears in the definition of $f$ can be explicitly computed. Indeed,

$$
\begin{aligned}
f(w) & =\frac{1}{2 \pi} \int_{0}^{2 \pi} \int_{0}^{\infty} \frac{\rho \cos \theta}{w \rho \cos \theta+\rho \sin \theta} e^{-\rho^{2} / 2} \rho d \rho d \theta \\
& =\frac{1}{2 \pi} \int_{0}^{2 \pi} \frac{d \theta}{w+\tan \theta}
\end{aligned}
$$

and the substitution $u=\tan \theta$ can then be used to rewrite this integral as

$$
f(w)=\frac{1}{\pi} \int_{-\infty}^{\infty} \frac{1}{(w+u)\left(1+u^{2}\right)} d u
$$

which is easily solved using the calculus of residues. The final result is that

$$
f(w)= \begin{cases}\frac{1}{w+i}, & \operatorname{Im}(w)>0 \\ \frac{1}{w-i}, & \operatorname{Im}(w)<0\end{cases}
$$

Recalling the definition of $\delta$ and $\gamma$, we see that

$$
\frac{\gamma}{\delta}=\frac{l_{21}}{l_{22}}-i \frac{l_{11}}{l_{22}}
$$

In general, $l_{11}$ and $l_{22}$ are just nonnegative. However, it is not hard to show that they are both strictly positive whenever $z$ has a nonzero imaginary part. Hence, $\gamma / \delta$ lies in the lower half-plane, $\delta / \gamma$ lies in the upper half-plane, and

$$
\begin{aligned}
& F(z)=\frac{\alpha}{\delta} \frac{1}{\frac{\gamma}{\delta}-i}+\frac{\beta}{\gamma} \frac{1}{\frac{\delta}{\gamma}+i} \\
& =\frac{i \alpha+\beta}{i \gamma+\delta}=\frac{l_{32}-l_{41}+i\left(l_{31}+l_{42}\right)}{-l_{21}+i\left(l_{11}+l_{22}\right)} \text {. }
\end{aligned}
$$


At this point, we need explicit formulas for the elements of the Cholesky factor $L$. From (2.9) and (2.10), we see that

$$
\begin{array}{ll}
a^{T} a=l_{11}^{2}, & \\
b^{T} a=l_{21} l_{11}, & b^{T} b=l_{21}^{2}+l_{22}^{2}, \\
c^{T} a=l_{31} l_{11}, & c^{T} b=l_{31} l_{21}+l_{32} l_{22}, \\
d^{T} a=l_{41} l_{11}, & d^{T} b=l_{41} l_{21}+l_{42} l_{22} .
\end{array}
$$

Solving these equations in succession, we get

$$
\begin{array}{ll}
l_{11}=\frac{a^{T} a}{\sqrt{a^{T} a}}, & \\
l_{21}=\frac{b^{T} a}{\sqrt{a^{T} a}}, & l_{22}=\frac{\left(a^{T} a\right)\left(b^{T} b\right)-\left(b^{T} a\right)^{2}}{\sqrt{a^{T} a} R}, \\
l_{31}=\frac{c^{T} a}{\sqrt{a^{T} a}}, & l_{32}=\frac{\left(a^{T} a\right)\left(c^{T} b\right)-\left(c^{T} a\right)\left(b^{T} a\right)}{\sqrt{a^{T} a} R}, \\
l_{41}=\frac{d^{T} a}{\sqrt{a^{T} a}}, & l_{42}=\frac{\left(a^{T} a\right)\left(d^{T} b\right)-\left(d^{T} a\right)\left(b^{T} a\right)}{\sqrt{a^{T} a} R},
\end{array}
$$

where

$$
R=\sqrt{\left(a^{T} a\right)\left(b^{T} b\right)-\left(b^{T} a\right)^{2}} .
$$

Substituting these expressions into (2.12) and simplifying, we see that

$$
F(z)=\frac{-d^{T} a+i c^{T} a-i\left(a^{T} a\left(-d^{T} b+i c^{T} b\right)-\left(-d^{T} a+i c^{T} a\right) b^{T} a\right) / R}{-b^{T} a+i a^{T} a+i R} .
$$

Recalling the definitions of $a_{j}, b_{j}, c_{j}$, and $d_{j}$ given in (2.8), it is easy to check that the following identities hold:

$$
\begin{aligned}
a^{T} a & =+\frac{1}{4}\left(A_{0}+2 B_{0}+\overline{A_{0}}\right), & & \\
b^{T} a & =-\frac{l}{4}\left(A_{0}-\overline{A_{0}}\right), & b^{T} b & =-\frac{1}{4}\left(A_{0}-2 B_{0}+\overline{A_{0}}\right), \\
c^{T} a & =+\frac{1}{4}\left(A_{1}+2 B_{1}+\overline{A_{1}}\right), & & c^{T} b=-\frac{1}{4}\left(A_{1}-\overline{A_{1}}\right), \\
d^{T} a & =-\frac{l}{4}\left(A_{1}-\overline{A_{1}}\right), & d^{T} b & =-\frac{1}{4}\left(A_{1}-2 B_{1}+\overline{A_{1}}\right) .
\end{aligned}
$$

Plugging these expressions into (2.13) and simplifying, we get that

$$
F(z)=\frac{A_{1}+B_{1}+\left(A_{0} B_{1}+B_{0} B_{1}-A_{1} B_{0}-\bar{A}_{0} A_{1}\right) / D_{0}}{A_{0}+B_{0}+D_{0}},
$$

where $D_{0}$ is given in (1.2). It turns out that a further simplification occurs if we make the denominator real by the usual technique of multiplying and dividing by its complex conjugate. We omit the algebraic details except to mention that a factor of $A_{0}+2 B_{0}+\bar{A}_{0}$ cancels out from the numerator and denominator, 
leaving us with

$$
F(z)=\frac{B_{1} D_{0}+B_{0} B_{1}-\overline{A_{0}} A_{1}}{D_{0}\left(B_{0}+D_{0}\right)},
$$

or, expanding out $D_{0}^{2}$,

$$
F(z)=\frac{B_{1} D_{0}+B_{0} B_{1}-\overline{A_{0}} A_{1}}{B_{0} D_{0}+B_{0}^{2}-\bar{A}_{0} A_{0}} .
$$

The expression in (2.15) is as simple as possible, whereas (2.16) illustrates a certain parallelism between the numerator and denominator.

Now consider a point $x$ on the real axis. On the reals, $A_{k}=B_{k}, k=0,1$, and so $D_{0}=0$. Hence, the right-hand side in (2.15) is an indeterminate form. To analyze the limiting behavior of $F$ near the real axis, we first divide the numerator and denominator by $D_{0}$ :

$$
F=\frac{B_{1}+\frac{B_{0} B_{1}-\bar{A}_{0} A_{1}}{D_{0}}}{B_{0}+D_{0}} .
$$

Now, only the ratio in the numerator is indeterminate. To study it, we write $z=r e^{i \theta}$ and investigate the numerator and denominator of this ratio when $\theta$ is small. From the definitions, we see that

$$
\begin{aligned}
B_{0} B_{1}-\bar{A}_{0} A_{1} & =\sum_{j, k} k r^{2(k+j)}\left(1-e^{2(k-j) \theta i}\right) \\
& =\sum_{j, k} k r^{2(k+j)} 2(j-k) \theta i+o(\theta) \\
& =2 \theta i\left(B_{1}^{2}-B_{0} B_{2}\right)+o(\theta)
\end{aligned}
$$

and

$$
\begin{aligned}
B_{0}^{2}-\left|A_{0}\right|^{2} & =\sum_{j, k} r^{2(k+j)}\left(1-e^{2(k-j) \theta i}\right) \\
& =-\sum_{j, k} r^{2(k+j)} 2(k-j)^{2} \theta^{2} i^{2}+o\left(\theta^{2}\right) \\
& =4 \theta^{2}\left(B_{0} B_{2}-B_{1}^{2}\right)+o\left(\theta^{2}\right)
\end{aligned}
$$

Hence, recalling that $D_{0}=\sqrt{B_{0}^{2}-\left|A_{0}\right|^{2}}$, we see that

$$
\begin{aligned}
\frac{B_{0} B_{1}-\bar{A}_{0} A_{1}}{D_{0}} & =\operatorname{sgn}(\theta) i \frac{B_{1}^{2}-B_{0} B_{2}+o(\theta)}{\left(B_{0} B_{2}-B_{1}^{2}+o\left(\theta^{2}\right)\right)^{1 / 2}} \\
& =-\operatorname{sgn}(\theta) i\left(B_{0} B_{2}-B_{1}^{2}\right)^{1 / 2}+o(\theta) .
\end{aligned}
$$

Combining (2.17) and (2.20), we get the desired limits expressing the jump discontinuity on the real axis.

Proof of Theorem 1.1. Without loss of generality, it suffices to consider regions $\Omega$ that are either regions that do not intersect the real axis or polar rectangles that do intersect the real axis. We begin by considering a region $\Omega$ that does not 
intersect the real axis. Applying Stokes' theorem to the expression for $\mathbf{E} \nu_{n}(\Omega)$ given in Proposition 2.1, we see that

$$
\mathbf{E} \nu_{n}(\Omega)=\frac{1}{\pi} \int_{\Omega} \frac{1}{z} \frac{\partial}{\partial \bar{z}} F(z, \bar{z}) d x d y .
$$

Note that we are now writing $F(z, \bar{z})$ to emphasize the fact that $F$ depends on both $z$ and $\bar{z}$. Letting the dagger symbol stand for the derivative with respect to $\bar{z}$, we see from Lemma 2.2 that

$$
\begin{aligned}
\frac{\partial F}{\partial \bar{z}}= & \left\{\left(B_{0} D_{0}+B_{0}^{2}-\bar{A}_{0} A_{0}\right)\left(B_{1}^{\dagger} D_{0}+B_{1} D_{0}^{\dagger}+B_{0}^{\dagger}+B_{0} B_{1}^{\dagger}-\bar{A}_{0}^{\dagger} A_{1}\right)\right. \\
& \left.-\left(B_{1} D_{0}+B_{0} B_{1}-\bar{A}_{0} A_{1}\right)\left(B_{0}^{\dagger} D_{0}+B_{0} D_{0}^{\dagger}+2 B_{0} B_{0}^{\dagger}-\bar{A}_{0}^{\dagger} A_{0}\right)\right\} \\
& /\left(B_{0} D_{0}+B_{0}^{2}-\left|A_{0}\right|^{2}\right)^{2} .
\end{aligned}
$$

From their definitions, it is easy to verify that

$$
B_{0}^{\dagger}=\frac{B_{1}}{\bar{z}}, \quad \bar{A}_{0}^{\dagger}=\frac{2 \bar{A}_{1}}{\bar{z}}, \quad B_{1}^{\dagger}=\frac{B_{2}}{\bar{z}} .
$$

Recalling that $D_{0}=\sqrt{B_{0}^{2}-|A|^{2}}$, we get that

$$
D_{0}^{\dagger}=\frac{B_{0} B_{1}-A_{0} \bar{A}_{1}}{\bar{z} D_{0}} .
$$

Substituting these formulas for the derivatives into the expression given above for $\partial F / \partial \bar{z}$ and then engaging in tedious algebraic simplifications (a computer algebra package such as Mathematica would be useful here, but we confess that we did this by hand), we eventually arrive at the fact that $(\pi z)^{-1} \partial F(z, \bar{z}) / \partial \bar{z}$ equals the expression given in Theorem 1.1 for $h_{n}$.

Now consider a polar rectangle that covers a portion of the real axis. In other words, let $\Omega$ be the angular interval $(-\theta, \theta)$ crossed with a radial interval $\left(r_{0}, r_{1}\right)$ (we have assumed without loss of generality that the polar rectangle does not intersect the negative part of the real axis). Writing down the contour integral for $\mathbf{E} \nu_{n}(\Omega)$ given by Proposition 2.1 and letting $\theta$ tend to 0 , we see that

$$
\mathbf{E} \nu_{n}\left(\left(r_{0}, r_{1}\right)\right)=\frac{1}{2 \pi i} \int_{r_{0}}^{r_{1}} \frac{F(r-)-F(r+)}{r} d r,
$$

where $\nu_{n}\left(\left(r_{0}, r_{1}\right)\right)$ denotes the number of zeros in the interval $\left(r_{0}, r_{1}\right)$ of the real axis and

$$
F(r-)=\lim _{z \rightarrow r: \operatorname{Im}(z)<0} F(z) \quad \text { and } \quad F(r+)=\lim _{z \rightarrow r: \operatorname{Im}(z)>0} F(z) .
$$

Hence, from Lemma 2.2, we see that

$$
g_{n}(r)=\frac{1}{2 \pi i} \frac{F(r-)-F(r+)}{r}=\frac{\sqrt{B_{0} B_{2}-B_{1}^{2}}}{\pi r B_{0}} .
$$

This completes the proof. 


\section{LIMITING INTENSITY}

In this section we study the limits obtained by letting $n$ tend to infinity. As mentioned in the introduction, these limits can be viewed as intensity functions for the limiting random power series. We begin by proving Theorem 1.2.

Proof of Theorem 1.2. The sums used to define the functions $B_{k}$ and $A_{k}$ can be summed explicitly. Recalling the functions $A$ and $B$ defined by (1.5) and (1.4), respectively, we have

$$
\begin{aligned}
B_{0}= & B-|z|^{2 n} B, \\
B_{1}= & |z|^{2} B^{2}-\left(n|z|^{2 n} B+|z|^{2 n+2} B^{2}\right), \\
B_{2}= & |z|^{2}\left(1+|z|^{2}\right) B^{3} \\
& -\left(n^{2}|z|^{2 n} B+2 n|z|^{2 n+2} B^{2}+|z|^{2 n+2}\left(1+|z|^{2}\right) B^{3}\right), \\
A_{0}= & A-z^{2 n} A, \\
A_{1}= & z^{2} A^{2}-\left(n z^{2 n} A+z^{2 n+2} A^{2}\right) .
\end{aligned}
$$

For $|z|<1$, we have the following limits:

$$
\begin{aligned}
& \lim _{n \rightarrow \infty} B_{0}=B, \\
& \lim _{n \rightarrow \infty} B_{1}=|z|^{2} B^{2}, \\
& \lim _{n \rightarrow \infty} B_{2}=|z|^{2}\left(1+|z|^{2}\right) B^{3}, \\
& \lim _{n \rightarrow \infty} A_{0}=A, \\
& \lim _{n \rightarrow \infty} A_{1}=z^{2} A^{2} .
\end{aligned}
$$

Using these limits, it is easy to check that

$$
\lim _{n \rightarrow \infty}\left(B_{2} B_{0}^{2}-B_{0} B_{1}^{2}\right)=|z|^{2} B^{5}
$$

and

$$
\begin{gathered}
\lim _{n \rightarrow \infty}\left(-B_{2}\left|A_{0}\right|^{2}-B_{0}\left|A_{1}\right|^{2}+B_{1}\left(A_{0} \bar{A}_{1}+\bar{A}_{0} A_{1}\right)\right) \\
\quad=-|z|^{2} B|A|^{2}\left(B^{2}+|\bar{z} B-z A|^{2}\right) .
\end{gathered}
$$

Then, using the definitions of $A, B$, and $D$, we see that

$$
|\bar{z} B-z A|^{2}=D^{2} \text {. }
$$

Substituting these ingredients into the expression given in Theorem 1.1 for $h_{n}$ and simplifying, we find that

$$
\lim _{n \rightarrow \infty} h_{n}=\frac{|z|^{2} B^{5}-|z|^{2} B|A|^{2}\left(B^{2}+|\bar{z} B-z A|^{2}\right)}{\pi|z|^{2} D^{3}}=\frac{B D}{\pi} .
$$

The case $|z|>1$ is more tedious (and less interesting!). We can either refer to the inversion symmetry formula (1.8) or we can compute in a manner similar to the $|z|<1$ case. Opting for the detailed computation, we note that this 
time we must keep track of terms involving the three highest orders: $n^{2}|z|^{2 n}$, $n|z|^{2 n}$, and $|z|^{2 n}$. Then we compute the numerator of the right-hand factor in the expression given in Theorem 1.1 for $h_{n}$ and we discover that the two highest order terms cancel out, leaving terms of the order $|z|^{2 n}$ as the highest remaining order. Indeed,

$$
\begin{gathered}
B_{2} B_{0}^{2}-B_{0} B_{1}^{2}=-|z|^{6 n}|z|^{2} B^{5}+o\left(|z|^{6 n}\right), \\
-B_{2}\left|A_{0}\right|^{2}-B_{0}\left|A_{1}\right|^{2}+B_{1}\left(A_{0} \bar{A}_{1}+\bar{A}_{0} A_{1}\right) \\
=|z|^{6 n}|z|^{2} B|A|^{2}\left(B^{2}+|\bar{z} B-z A|^{2}\right)+o\left(|z|^{6 n}\right),
\end{gathered}
$$

and

$$
D_{0}^{2}=|z|^{4 n} D^{2}+o\left(|z|^{4 n}\right) .
$$

Hence, the numerator and denominator of the right-hand factor both have $|z|^{6 n}$ as their highest order terms, so this factor can be canceled. For the left-hand factor, both numerator and denominator have $|z|^{4 n}$ as their highest order terms. Eliminating these and passing to the limit, we get

$$
\lim _{n \rightarrow \infty} h_{n}=\frac{-B D}{\pi} .
$$

Bearing in mind that $B>0$ for $|z|<1$ and $B<0$ for $|z|>1$, we see that (3.11) and (3.12) can be combined to give the formula asserted for $h$.

The same method is used to find $\lim _{n \rightarrow \infty} g_{n}$. Indeed, for $|z|<1$, we use (3.6), (3.7), and (3.8) to see that

$$
\lim _{n \rightarrow \infty} g_{n}=\frac{1}{\pi} B .
$$

For $|z|>1$, we retain the three highest order terms from (3.1), (3.2), and (3.3). As before the two highest order terms cancel out in the expression for $B_{0} B_{2}-B_{1}^{2}$, leaving us with

$$
B_{0} B_{2}-B_{1}^{2}=|z|^{4 n}|z|^{2} B^{4}+o\left(|z|^{4 n}\right) .
$$

From this expression, we easily see that

$$
\lim _{n \rightarrow \infty} g_{n}=-\frac{1}{\pi} B,
$$

which completes the proof.

\section{ASYMPTOTICS FOR THE NUMBER OF ROOTS}

In this section we derive limiting expressions for the expected number of zeros in disks and sectors. We start with the disk, $B(r)$, of radius $r$ centered at 0 .

Theorem 4.1. For $r<1$,

$$
\lim _{n \rightarrow \infty} \mathbf{E} \nu_{n}(B(r))=\frac{r^{2}}{1-r^{2}}+O(1) .
$$


Proof. From Proposition 2.1, we have that

$$
\begin{aligned}
\mathbf{E} \nu_{n}(B(r)) & =\frac{1}{2 \pi i} \int_{\partial B(r)} \frac{1}{z} F(z) d z \\
& =\frac{1}{2 \pi} \int_{0}^{2 \pi} F(z) d \theta .
\end{aligned}
$$

Now, applying the bounded convergence theorem, we see that

$$
\lim _{n \rightarrow \infty} \mathbf{E} \nu_{n}(B(r))=\frac{1}{2 \pi} \int_{0}^{2 \pi} \lim _{n \rightarrow \infty} F(z) d \theta
$$

and, using the expression given in (2.15) for $F$, we get that

$$
\lim _{n \rightarrow \infty} F(z)=\frac{|z|^{2} B^{2} D+B|z|^{2} B^{2}-\bar{A} z^{2} A^{2}}{(B+D) D} .
$$

Adding and subtracting $|z|^{2} B|A|^{2}$ from the numerator and using the fact that $|z|^{2} B=r^{2} /\left(1-r^{2}\right)$ on the contour of integration, we see that

$$
\lim _{n \rightarrow \infty} F(z)=\frac{r^{2}}{1-r^{2}}+\frac{|A|^{2}}{(B+D) D}\left(|z|^{2} B-z^{2} A\right) .
$$

From the definitions of $A, B$, and $D$, it is easy to check that

$$
|z|^{2} B-z^{2} A=\left(|z|^{2}-z^{2}\right) A B
$$

and that

$$
D=2|\operatorname{Im}(z)| B|A| \text {. }
$$

Hence, the second term on the right in (4.1) simplifies to

$$
\left(1-r^{2}\right) \frac{|A| A\left(r^{2}-z^{2}\right)}{2(1+2|\operatorname{Im}(z)||A|)|\operatorname{Im}(z)|}
$$

and so the proof will be complete if we show that the integral of this expression over the circle of radius $r$ is bounded. To this end, we note that the triangle inequality combined with the fact that $|\sin \theta|=\left|1-e^{2 i \theta}\right| / 2$ gives us

$$
\left|\int_{0}^{2 \pi} \frac{|A| A\left(r^{2}-z^{2}\right)}{2(1+2|\operatorname{Im}(z)||A|)|\operatorname{Im}(z)|} d \theta\right| \leq r \int_{0}^{2 \pi} \frac{1}{\left|1-r^{2} e^{2 i \theta}\right|^{2}} d \theta .
$$

The integral on the right-hand side can be integrated explicitly:

$$
\int_{0}^{2 \pi} \frac{1}{\left|1-r^{2} e^{2 i \theta}\right|^{2}} d \theta=\frac{2 \pi}{\left(1-r^{2}\right)\left(1+r^{2}\right)}
$$

Combining (4.4), (4.3), and (4.2), we get that the second term in (4.1) is bounded by $2 \pi r /\left(1+r^{2}\right)$. This completes the proof.

Theorem 4.2. For $s \geq 0$,

$$
\lim _{n \rightarrow \infty} \mathbf{E} \frac{1}{n} \nu_{n}\left(B\left(e^{-s / 2 n}\right)\right)=\frac{\left(1-e^{-s}(1+s)\right)}{s\left(1-e^{-s}\right)}=\frac{1}{2}-\frac{s}{12}+o(s) .
$$


Proof. We begin as we began the proof of the previous theorem with

$$
\mathbf{E} \nu_{n}(B(r))=\frac{1}{2 \pi} \int_{0}^{2 \pi} F(z) d \theta,
$$

where

$$
F(z)=\frac{B_{1}+\frac{B_{0} B_{1}-\bar{A}_{0} A_{1}}{D_{0}}}{B_{0}+D_{0}}
$$

and this time

$$
r=e^{-s / 2 n}
$$

and

$$
z=r e^{i \theta} .
$$

The theorem then follows from the dominated convergence theorem and the following easy-to-derive asymptotic formulas (the last two holding everywhere except $\theta=0$ and $\theta=\pi$ ):

$$
\begin{aligned}
& \lim _{n \rightarrow \infty} \frac{1}{n} B_{0}=\lim _{n \rightarrow \infty} \frac{1}{n} D_{0}=\left(1-e^{-s}\right) / s, \\
& \lim _{n \rightarrow \infty} \frac{1}{n^{2}} B_{1}=\left(1-e^{-s}(1+s)\right) / s^{2}, \\
& \lim _{n \rightarrow \infty} \frac{1}{n} A_{0}=\lim _{n \rightarrow \infty} \frac{1}{n} A_{1}=0 .
\end{aligned}
$$

Let $S\left(\theta_{0}, \theta_{1}\right)$ denote the sector of the complex plane consisting of all points whose argument lies between $\theta_{0}$ and $\theta_{1}$. The next theorem shows that asymptotically the zeros are uniformly distributed in argument. Of course, the $2 / \pi \log n$ real zeros disappear since we are normalizing by dividing by $n$.

Theorem 4.3. For each sector $S\left(\theta_{0}, \theta_{1}\right)$ that does not intersect the real axis,

$$
\lim _{n \rightarrow \infty} \frac{1}{n} \mathbf{E} \nu_{n}\left(S\left(\theta_{0}, \theta_{1}\right)\right)=\frac{\theta_{1}-\theta_{0}}{2 \pi} .
$$

Šparo and Šur [14] obtained the analogous result for complex Gaussians using a very general and elegent result of Erdös and Turán [4] ( Theorem 8.1).

Proof. According to (1.7), it suffices to prove the analogous limit for the intersection, $P\left(\theta_{0}, \theta_{1}\right)$, of $S\left(\theta_{0}, \theta_{1}\right)$ with the unit disk. By Proposition 2.1,

$$
\mathbf{E} \nu_{n}\left(P\left(\theta_{0}, \theta_{1}\right)\right)=\frac{1}{2 \pi i} \int_{0}^{1} \frac{1}{r}\left(F\left(r e^{i \theta_{0}}\right)-F\left(r e^{i \theta_{1}}\right)\right) d r+\frac{1}{2 \pi} \int_{\theta_{0}}^{\theta_{1}} F\left(e^{i \theta}\right) d \theta,
$$

where $F$ is given by $(2.2)$. Note that the first integrand is bounded uniformly in $n$ even at $r=0$. To see this, we take note of the following limits:

$$
\begin{gathered}
\lim _{r \rightarrow 0} B_{0}=\lim _{r \rightarrow 0} A_{0}=1, \\
\lim _{r \rightarrow 0} D_{0}=0, \\
\lim _{r \rightarrow 0} \frac{1}{r} B_{1}=1,
\end{gathered}
$$


and

$$
\lim _{r \rightarrow 0} \frac{1}{r} \frac{B_{0} B_{1}-\bar{A}_{0} A_{1}}{D_{0}}=-\frac{e^{i \theta}}{2 \sin \theta} .
$$

This last limit is easy to obtain from expressions (2.18) and (2.19). Hence, from (2.17) we see that

$$
\lim _{r \rightarrow 0} \frac{1}{r} F\left(r e^{i \theta}\right)=1-\frac{e^{i \theta}}{2 \sin \theta}
$$

and so we can interchange limit and integration to see that in the limit $1 / n$ times the first integral vanishes.

To study the limiting behavior of $1 / n$ times the second integral, we first rewrite $F$ as

$$
F=\frac{B_{1}}{D_{0}}-\frac{\bar{A}_{0} A_{1}}{D_{0}\left(B_{0}+D_{0}\right)}
$$

Now, for $z=e^{i \theta}$,

$$
B_{0}=n, \quad B_{1}=\frac{n(n-1)}{2}, \quad \text { and } \quad D_{0}=\sqrt{n^{2}-\left|A_{0}\right|^{2}}
$$

Since $A_{0}$ and $A_{1}$ are bounded on $P\left(\theta_{0}, \theta_{1}\right)$, it follows that

$$
\lim _{n \rightarrow \infty} \frac{1}{n} \frac{B_{1}}{D_{0}}=\frac{1}{2}
$$

and

$$
\lim _{n \rightarrow \infty} \frac{1}{n} \frac{\overline{A_{0}} A_{1}}{D_{0}\left(B_{0}+D_{0}\right)}=0
$$

Therefore,

$$
\lim _{n \rightarrow \infty} \frac{1}{n} F\left(e^{i \theta}\right)=\frac{1}{2}
$$

and so the dominated convergence theorem implies that

$$
\lim _{n \rightarrow \infty} \frac{1}{n} \mathbf{E} \nu_{n}\left(P\left(\theta_{0}, \theta_{1}\right)\right)=\frac{\theta_{1}-\theta_{0}}{4 \pi} .
$$

This completes the proof.

\section{NUMERICAL COMPUTATION}

In this section, we describe the computer program used to produce Figures 1 to 5. Each figure shows two plots. On the left is a grey-scale plot of the intensity functions $g_{n}$ and $h_{n}$. On the right is a plot of 20,000 zeros obtained by generating random polynomials and explicitly finding their zeros.

The intensity plots appearing on the left were produced by partitioning the given square domain into a $256 \times 256$ grid of "pixels" (i.e., small squares) and computing the intensity function in the center of each pixel. The grey-scale was computed by assigning white to the pixel with the smallest value and black to the pixel with the largest value and then linearly interpolating all values in between. This grey-scale computation was performed separately for $h_{n}$ and for $g_{n}$ (which appears only on the $x$-axis) and so no conclusions should be drawn comparing the intensity shown on the $x$-axis with that shown off of it.

Of course, the intensity function $g_{n}$ is one-dimensional and therefore it would be natural (and more informative) to make separate plots of values of 

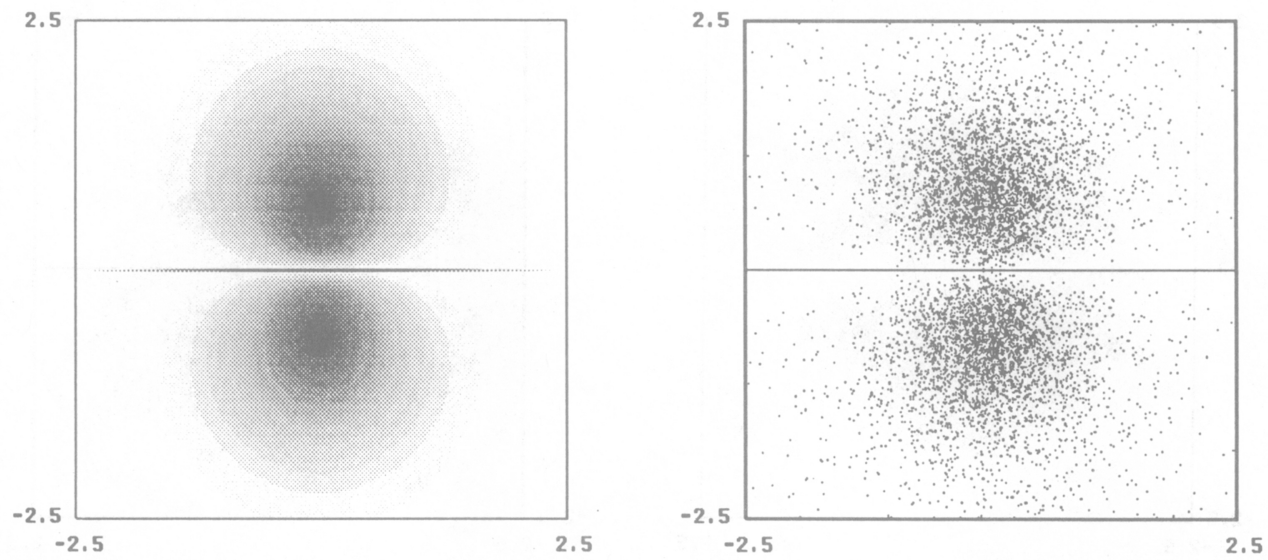

FigURE 1. Random quadratics $(n=3)$. In this figure and the next several, the left-hand plot is a grey-scale image of the intensity functions $h_{n}$ and $g_{n}$ (which is concentrated on the $x$-axis). The right-hand plot shows 20,000 roots from randomly generated polynomials. Subsequent captions give more information pertaining to each of these figures.
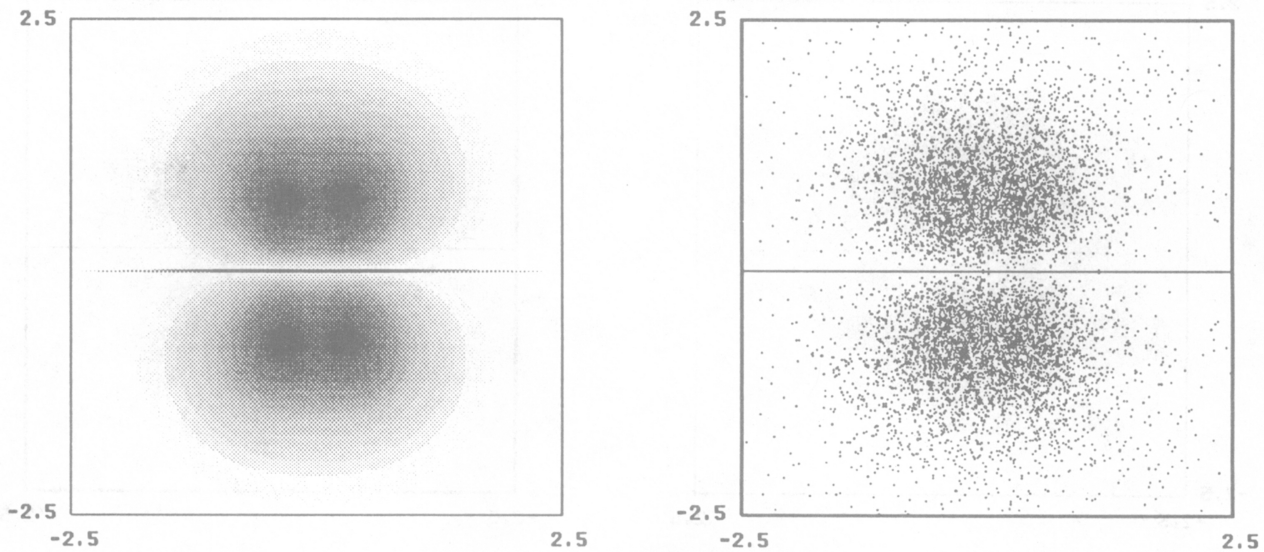

FiguRE 2. Random cubics $(n=4)$. Note that, for the left-hand plots, the grey-scales for $h_{n}$ and $g_{n}$ are produced separately and in such a way that both use the full range from white to black.

$g_{n}$ verses $x$, but such plots appear in many places (see, e.g., [8]) and so it seemed unnecessary to produce them here. Suffice it to say that $g_{n}$ is a density whose mass lies primarily near \pm 1 .

The 20,000 zeros plotted on the right were produced by a novel root-finding algorithm, which we shall describe briefly. Given a polynomial $P$, the algorithm that finds its zeros in a given square $R$ is recursive. Let $S$ denote the smallest disk that contains $R$. Using the argument principle, we can compute the number of zeros in $S$ :

$$
\nu(S)=\frac{1}{2 \pi i} \int_{\partial S} \frac{P^{\prime}(z)}{P(z)} d z .
$$



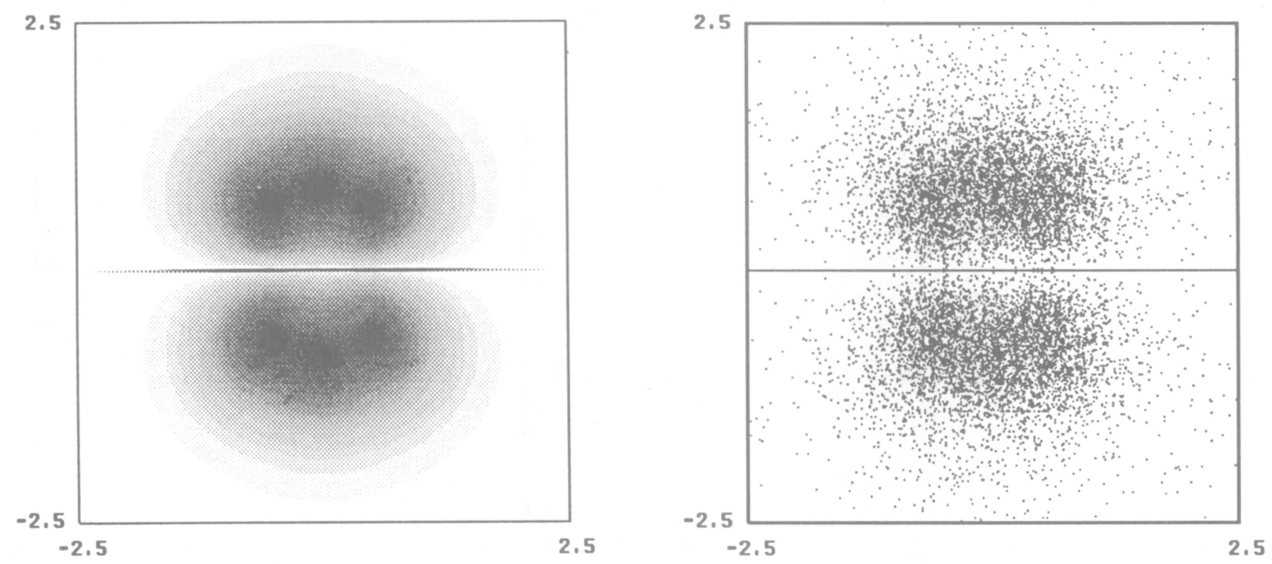

FIgURE 3. Random quartics $(n=5)$. Note that, for the righthand plots, most if not all of the "pixels" on the $x$-axis have been hit by at least one root. A more accurate image for the $x$-axis would have been obtained had we used a grey-scale to indicate how many times each pixel on the $x$-axis was hit.
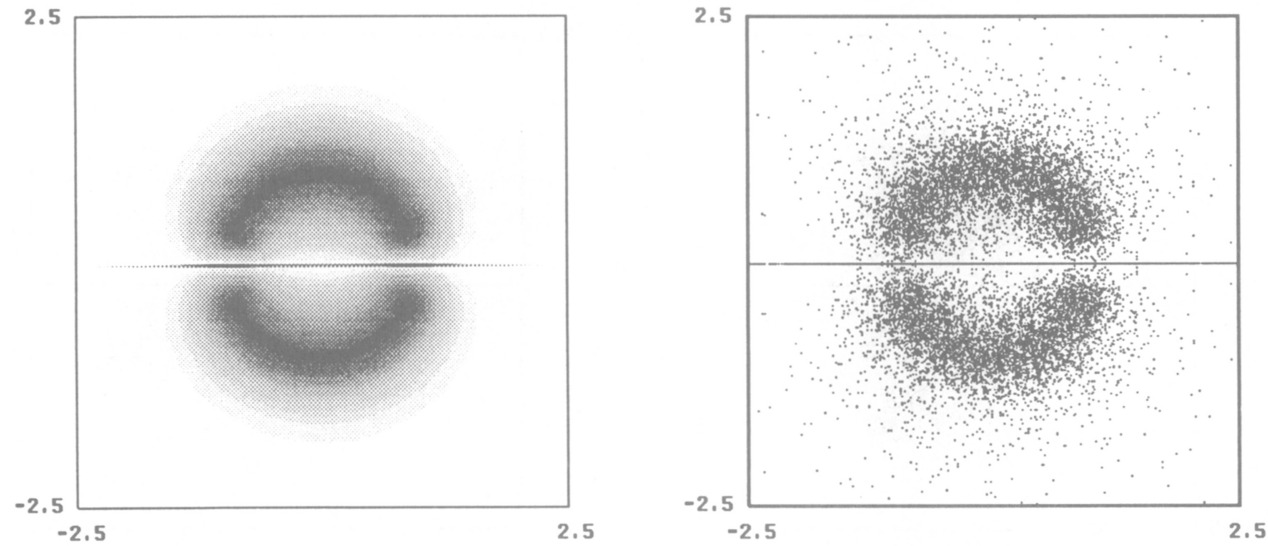

FIGURE 4. $n=10$.

If $\nu(S)$ is zero, then we stop since there are then no zeros in $R$. Otherwise, we compute the sum of the zeros using the following analogue of the argument principle:

$$
\sigma(S)=\sum_{i: z_{i} \in S} z_{i}=\frac{1}{2 \pi i} \int_{\partial S} \frac{z P^{\prime}(z)}{P(z)} d z .
$$

Here, the $z_{i}$ 's denote the zeros of $P$. The ratio

$$
\alpha(S)=\sigma(S) / \nu(S)
$$

is then the barycenter of the zeros contained in $S$ (counting multiplicities). Next, let $B$ be a disk of very small radius centered at $\alpha(S)$, and again using 

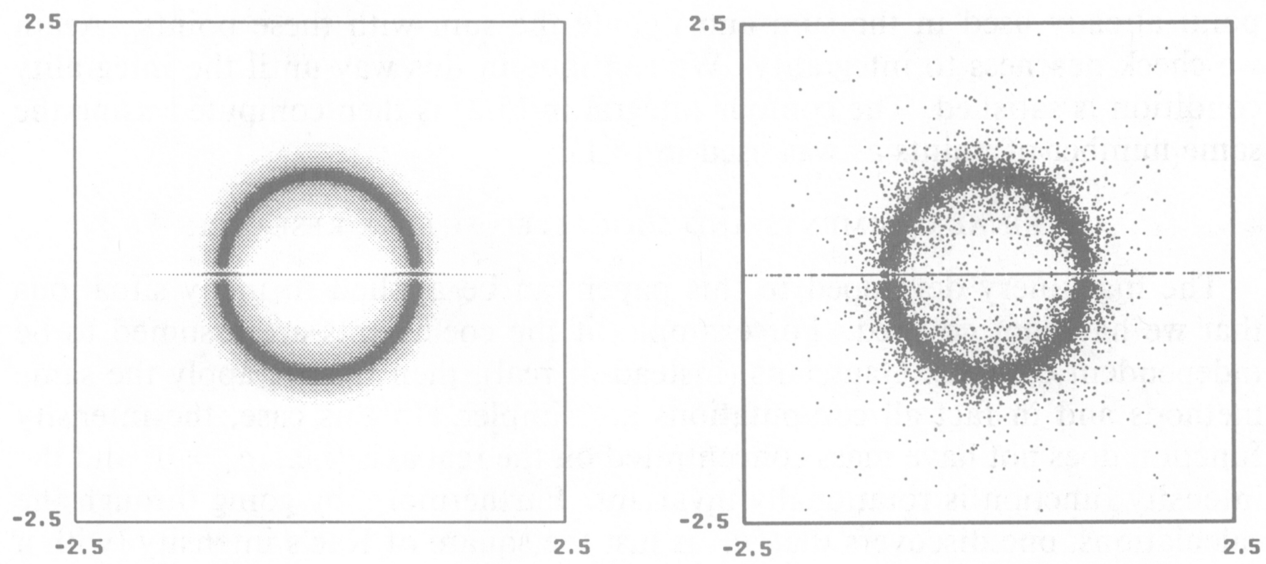

FIGURE 5. $n=36$.

2.5

$$
\begin{array}{r}
-2.5 \\
-2.5
\end{array}
$$$$
2.5
$$

FIGURE 6. Limiting intensity (using a logarithmic grey-scale).

the argument principle count the number of zeros in this disk. If this number equals $\nu(S)$, then there is only one zero in $S:$ it is at $\alpha(S)$ and has multiplicity $\nu(S)$. Hence, again we stop (but first, we check whether the zero is in fact in $R$ and if it is, we add it to our list of zeros found so far). In all other cases, there are still two or more zeros to be found and so we partition the square into four subsquares and recursively call this same procedure on those subsquares. Except for discussing how to compute the contour integrals, this is a complete description of the algorithm used to produce the right-hand plots.

The contour integrals in (5.1) and (5.2) are computed as follows. First we compute $\nu(S)$. To do this, we begin by simply using a discrete approximation to the integral consisting of eight points spread equally around the boundary of $S$. Then we check to see if $\nu(S)$ is close to being an integer. If it is, we are done. If it is not, then we spread eight more points midway between each 
point already used in the sum and update the sum with these points. Again we check nearness to integrality. We continue in this way until the integrality condition is satisfied. The contour integral in (5.2) is then computed using the same number of points as was used in (5.1).

\section{FinAl COMMENTS AND SUGGESTED FUTURE RESEARCH}

The machinery developed in this paper can be applied in many situations that we have not covered. For example, if the coefficients are assumed to be independent complex Gaussians (instead of real), then we can apply the same methods and in fact all computations are simpler. In this case, the intensity function does not have mass concentrated on the real axis (i.e., $g_{n}=0$ ) and the intensity function is rotationally invariant. Furthermore, by going through the calculations, one discovers that $h_{n}$ is just the square of Kac's intensity (with a different lead constant):

$$
h_{n}=\frac{B_{0} B_{2}-B_{1}^{2}}{\pi|z|^{2} B_{0}^{2}} .
$$

The classic paper of Hammersley [5] covers this case (among others). Also, the random power series version was considered in [3].

In the case of real polynomials, Kac's formula has been extended to other distributions besides the normal law for the coefficients as well as in many other directions (see [2]). In view of the interest in zeros indicated by the enormous literature on the real zeros it is amazing that the simple results given here for the complex zeros were not found earlier. One extension [10,11] considers replacing the normal distribution by symmetric stable distributions, $S(\alpha), 0<\alpha<2$, and obtains that the expected number of real zeros is asymptotic to $c(\alpha) \log n$ as $n$ tends to infinity. The coefficient $c(\alpha)$ decreases from 1 to $2 / \pi$ as $\alpha$ goes from 0 to 2 and is given by an explicit formula. Stevens [13] showed that if the coefficients are independent and merely in the domain of attraction of the normal law, then the expected number of real zeros follows the same asymptotic, $\frac{2}{\pi} \log n$, and Ibragimov and Maslova [6] proved the corresponding result for the case when the coefficients are independent and in the domain of attraction of the stable law with parameter $1<\alpha<2$.

For the complex case, we do not see how to extend any of our results to a domain of attraction statements. Moreover, we do not know what the change will be for $h_{n}(\alpha, x)$ if the coefficients are symmetric stable instead of normal. The techniques of $[10,11]$ do not seem to exterd easily to the complex case. Needless to say, the same holds for coefficients merely within the domain of attraction of a symmetric stable law. However, we conjecture that the domain of attraction cases follow the same asymptotics as the corresponding stable law, in particular if $\alpha=2$.

Let $\rho_{j}$ denote the norm of the zero of $P_{n}(z)$ that is inside the unit circle and the $j$-th closest to the unit circle. The results of this paper indicate that $\rho_{j}=1-X_{j} / n^{2}$, where the random variables $X_{1}, X_{2}, \ldots$ form a standard Poisson process. Similarly, the Kac formula for the real zeros suggests a similar conjecture except with a division by $n$ instead of $n^{2}$. If these two statements are correct, then the complex zeros are much closer to the unit circle than the real zeros are to \pm 1 . 
Assuming that the above conjectures are correct, there is a speculative corollary/application to Littlewood's problem of studying the behavior as $n$ tends to infinity of the random variable defined as

$$
m_{n}=\inf _{0 \leq \theta \leq 2 \pi}\left|P_{n}\left(e^{i \theta}\right)\right|
$$

i.e., the infimum on the unit circle of $P_{n}(z)$. Here, the coefficients are assumed to be independent symmetric \pm 1 random variables. Konyagin [9] gave a new estimate for $m_{n}$ by a rather complicated argument. We cannot obtain his delicate estimate by our methods, but there is at least some hope that this can be done provided that some further developments can be carried out. One would have to prove that the conjecture that the nearest complex zero to the unit circle is within $O\left(1 / n^{2}\right)$ holds not only for normal coefficients but for \pm 1 coefficients. As pointed out privately by A. M. Odlyzko, it would then follow from Bernstein's theorem that an alternate proof to Konyagin's estimates would be obtainable.

\section{ACKNOWLEDGMENT}

The results of this paper and our resulting speculations on finding an alternate proof based on studying the zeros of $P_{n}(z)$ near the unit circle were stimulated by a talk by S.V. Konyagin. In the course of these speculations, the study of the zeros themselves began to be even more interesting to us than the application to Littlewood's problem. The application will have to await further inspiration and another paper (possibly by other authors).

We would also like to acknowledge Ofer Zeitouni for carefully reading an early draft of the paper and suggesting some important algebraic simplifications.

\section{REFERENCES}

1. L.V. Ahlfors, Complex analysis, McGraw-Hill, New York, 1966.

2. A.T. Bharucha-Reid and M. Sambandham, Random polynomials, Academic Press, New York, 1986.

3. A. Edelman and E. Kostlan, How many zeros of a random polynomial are real?, Bull. Amer. Math. Soc. 32 (1985), 1-37.

4. P. Erdös and P. Turán, On the distribution of roots of polynomials, Ann. of Math. (2) 51 (1950), 105-119.

5. J. Hammersley, The zeros of a random polynomial, Proc. Third Berkeley Sympos. Math. Stat. Probability, vol. 2, 1956, pp. 89-111.

6. I. A. Ibragimov and N. B. Maslova, On the expected number of real zeros of random polynomials. I. Coefficients with zero means, Teor. Veroyatnost. i Primenen. 16 (1971), 229248.

7. M. Kac, On the average number of real roots of a random algebraic equation, Bull. Amer. Math. Soc. 49 (1943), 314-320, 938.

8. __ Probability and related topics in physical sciences, Interscience, London, 1959.

9. S. V. Konyagin, On minimum of modulus of random trigonometric polynomials with coefficients \pm 1 , Mat. Zametki 56 (1994), 80-101. (Russian) 
10. B. F. Logan and L. A. Shepp, Real zeros of random polynomials, Proc. London Math. Soc. (3) 18 (1968), 29-35.

11. 314.

12. S. O. Rice, Mathematical theory of random noise, Bell System Tech. J. 25 (1945), 46-156.

13. D. C. Stevens, The average and variance of the number of real zeros of random functions, Ph.D. Dissertation, New York University, New York, 1965.

14. D. I. Šparo and M.G. Sur, On the distribution of roots of random polynomials, Vestnik Moskov. Univ. Ser. I Mat. Mekh. (1962), 40-53.

at\&T Bell laboratories, Murray Hill, New Jersey 07974

E-mail address: las@research.att.com

Program in Statistics and Operations Research, Princeton University, Princeton, NEW JERSEY 08544

E-mail address: rvdb@princeton.edu 